\title{
Liberação miofascial na prevenção de lesão muscular: relato de caso
}

\author{
Julio Cesar Dias Junior*
}

Fisioterapeuta, membro da Sociedade Nacional de Fisioterapia Esportiva e Atividade Física - SONAFE

\section{Histórico do Artigo Recebido em: 03/03/2020 \\ Aceito em: 20/05/2020}

Palavras-chave: Liberação miofascial; prevenção de lesão; fáscia; liberação instrumental

\section{Keywords:}

Myofascial release; injury prevention; fascia; instrumental release

\begin{abstract}
RESUMO
A fáscia é uma estrutura formada de tecido conjuntivo, que envolve os músculos e pode sofrer aderências diminuindo o deslizamento dos tecidos. A liberação miofascial é uma técnica de pressão em partes do corpo que tende a melhorar a vascularização, maleabilidade, relaxamento dos tecidos, facilitando a mobilidade. O objetivo desta pesquisa foi testar a utilização da liberação miofascial na prevenção lesões musculares em um atleta de futebol amador. O relato de caso foi com um atleta de futebol amador, 36 anos, com lesões recorrentes em membros inferiores e apresentando grande desgaste físico e dores musculares, avaliado através de anamnese e exame físico, escalas: Borg e visual analógica para exaustão física e dor. Foi implementado a liberação miofascial instrumentada, uma vez na semana, por 60 minutos nos músculos dos membros inferiores. Os resultados apresentaram que após a terapia o atleta relatou melhora nas dores musculares, no cansaço físico e não apresentou mais lesões musculares. Alguns estudos mostram que não há melhora da força muscular após a prática da liberação miofascial, porém mostrou-se efetivo na melhora significativa da flexibilidade e consequentemente na recuperação funcional dos músculos. Concluí-se que neste caso, a liberação miofascial foi eficaz na melhora recuperação muscular, da flexibilidade, da força, prevenindo lesões.
\end{abstract}

\section{Myofascial release in the prevention of muscle injury: case report}

\begin{abstract}
The fascia is a structure formed of connective tissue, which surrounds the muscles and can suffer adhesions reducing the tissues sliding. Myofascial release is a pressure technique on parts of the body that tends to improve the tissue vascularity, suppleness, relaxation, facilitating mobility. The aim of this research was to test the use of myofascial release in preventing muscle injuries in an amateur soccer athlete. The case report was with an amateur soccer player, 36 years old, with recurrent injuries in the lower limbs and presenting great physical wear and muscle pain, assessed through anamnesis and physical examination, scales: Borg and visual analog for physical exhaustion and pain. Instrumented myofascial release was implemented, once a week, for 60 minutes in the lower limb muscles. The results showed that after therapy the athlete reported improvement in muscle pain, physical tiredness and did not present any more muscle injuries. Some studies show that there is no improvement in muscle strength after the practice of myofascial release, but it was effective in significantly improving flexibility and, consequently, in the functional recovery of muscles. It was concluded that in this case, the myofascial release was effective in improving muscle recovery, flexibility, strength, acting in the prevention of injuries.
\end{abstract}

\section{Introdução}

A fáscia é uma estrutura do corpo humano composta de tecido conjuntivo, que envolve os músculos, nervos, vasos sanguíneos e conecta as estruturas. Estes tecidos são resistentes e elásticos podendo sofrer adaptações e restrições, como: doenças; lesões; inatividade física; inflamação; aderência (1-3). Ela é subdividida em superficial e profunda, revestindo as fibras, fascículos e todo ventre muscular: endomísio; perimísio; epimísio. Estas estruturas são responsáveis pela conexão dos ossos e músculos do corpo, tornando-se importante na transmissão de força (tração / compressão) muscular $(4,5)$.

A doença mais comum é a síndrome dolorosa miofascial caracterizada por uma

\footnotetext{
*Autor correspondente: juliofisioterapia@yahoo.com.br (Dias Junior J.C.)
} 
disfunção neuromuscular local, apresentando bandas musculares tensas, desencadeando dor local ou irradiada. Sua prevalência é de difícil determinação pois os critérios diagnósticos são clínicos e dependem de uma avaliação minuciosa identificando os trigger points, acometendo indivíduos de ambos os sexos, atletas e não atletas acima dos 30 anos (6). A lesão mais comum que acomete a fáscia é a de esforço repetitivo (LER), dentre as mais conhecidas são as tendinites, tenossinovites e epicondilites, prevalecendo em trabalhadores no auge da produtividade e experiência profissional, com uma faixa etária de 30 a 40 anos (7).

Quando sobrecarregada, este tecido pode sofrer alterações, como desidratação, evoluindo para aderências fibrosas, diminuindo fluidez, desencadeando perda de flexibilidade, dor e diminuição da amplitude de movimento (ADM) (8). Mas sendo uma estrutura que possuí uma histerese: capacidade de receber carga, sofrer deformidade, alterando seu comprimento e ao cessar esta carga, o tecido se restabelece plenamente, a fáscia, pode receber intervenções manuais, efetuando uma pressão nessas regiões aderidas, para atingirmos este processo $(5,9,10)$.

A terapia manual agindo diretamente sobre o tecido conjuntivo, da-se o nome de liberação miofascial, podendo utilizar diversas técnicas para esta função $(2,11)$. Esticando, alterando o comprimento histológico, aliviando sintomas do encurtamento miofascial, contribuí-se para o ganho de ADM, alívio das dores e recomposição da normalidade e qualidade deste tecido $(3,12,13)$.

A auto liberação miofascial caracteriza-se pelo indivíduo realizar uma pressão sobre a fáscia, utilizando seu peso corporal ou algum dispositivo. Isso leva a um aumento do fluxo sanguíneo na região estimulada, melhorando a capacidade de deslizamentos destes tecidos e conseqüentemente a melhora da $\operatorname{ADM}(2,12)$.

Existem diversos recursos a serem utilizados para realizar a técnica de liberação miofascial, como por exemplo: crochetagem, raspadores, rolos, mãos, cotovelos, etc. O importante é a fundamentação de todo este princípio, promovendo aumento do fluxo sangüíneo para os músculos ativados, facilitando o trabalho neuromuscular, melhorando a oxigenação tecidual, auxiliando na melhora da flexibilidade e mobilidade (15). Contudo o cisalhamento promovido por esta intervenção gera uma extensão da fáscia onde favorecerá: melhora sintomática; ativação muscular; tonificação do tecido conjuntivo; melhora do desempenho atlético (2).

Este procedimento quando realizado antes da atividade, além de promover estas respostas fisiológicas pode ocorrer um fenômeno denominado tixotropia, no qual, o calor ou pressão no tecido, torna ele mais fluido e menos denso, aumentando a produção de óxido nítrico (vasodilatador) e mediadores inflamatórios (15). Além disso, o aumento da flexibilidade proporciona a melhora da ADM, muito importante para o desempenho e aptidão física, esportiva e da saúde em geral (2).

Autores relatam que a diminuição do condicionamento físico, da flexibilidade, prejudica a execução dos movimentos articulares colocando em risco esta estrutura e prejudicando a função da musculatura $(16,17)$. Nesta linha de pensamento, melhorar a flexibilidade, restaurar a amplitude de movimento funcional, aperfeiçoar o desempenho muscular e postural, a técnica de liberação miofascial, vem a ser um método de prevenção de lesões musculoesqueléticas $(18,19)$.

Outra técnica de terapia manual miofascial é a massoterapia, que pode trazer benefícios como: aumento da circulação; melhora do tônus muscular; equilíbrio das funções biológicas (relaxamento dos músculos; nervos; conforto articular); alivio das dores; alívio do estresse; além de prevenir doenças $(20,21)$.

Como a fáscia sofre essa inúmeras variações promovendo alterações significativas, este estudo de liberação miofascial tem por finalidade diminuir as adesões fibrosas nas redes 
fasciais, revertendo a perda de energia da estrutura envolvida após o estresse mecânico, evitando possíveis lesões e devolvendo a função para este atleta. Com a escassez de estudos relacionados, com a prevenção de lesões, justifica-se a importância desta pesquisa no cenário científico podendo obter uma ferramenta adicional com bons resultados na prática. O objetivo desta pesquisa foi testar a utilização da liberação miofascial na prevenção lesões musculares em um atleta de futebol amador.

\section{Métodos}

Foi selecionado um atleta de futebol amador, de uma cidade do interior do estado de São Paulo, com idade de 36 anos, 99 quilos, 1,79 metros, praticante da modalidade há mais de 20 anos. O mesmo sofreu uma grave lesão no joelho esquerdo (ruptura do ligamento cruzado anterior associado a lesão do menisco lateral), no dia 27 de janeiro de 2018. Foi submetido a uma cirurgia de reconstrução do ligamento cruzado anterior e meniscectomia parcial do menisco lateral, iniciando o processo de reabilitação em seguida e permanecendo em tratamento por 8 meses, até ser liberado para o retorno a prática esportiva pelo fisioterapeuta e pelo médico.

Um dos critérios para liberação da fisioterapia, foi o triple hop test distance e o crossover hop for distance, onde apresentou uma similaridade de $95 \%$ entre os membros, indicando um padrão de força muscular dentro da normalidade para retorno ao esporte (22).

Quando foi liberado, este atleta retornou a prática esportiva no mês de outubro de 2018, com o seguinte calendário: campeonatos de futebol amador 3 vezes por semana; treino de força muscular e condicionamento físico na academia 3 vezes por semana.

Após este retorno, no período de outubro/2018 à março/2019, o atleta apresentou muitas dores musculares nos membros inferiores, relatando uma sensação de cansaço, ocasionando 3 lesões musculares neste período, (uma no quadríceps femoral e duas nos isquiotibiais do membro inferior esquerdo) avaliadas através do diagnóstico funcional: história da moléstia atual (HMA) do paciente; teste de palpação; teste de contração da musculatura envolvida; teste de alongamento da musculatura envolvida; seguidos pela presença de hematoma na região, sugerindo ruptura dos vasos sangüíneos intra muscular e gap. Para avaliação das dores em membros inferiores foi utilizado a escala de Borg modificada (Figura 1): uma escala vertical quantificada de 0 a 10, onde 0 representa nenhum sintoma e 10 representa sintoma máximo de cansaço, que no caso deste estudo era muscular (23); e Escala Visual Analógica (EVA) (Figura 2): constituída por uma linha de $10 \mathrm{~cm}$, com os extremos "ausência de dor" com referência ao número 0 e "dor insuportável" no número 10, podendo apresentar expressões faciais na escala, como a usada nesta pesquisa (24). 


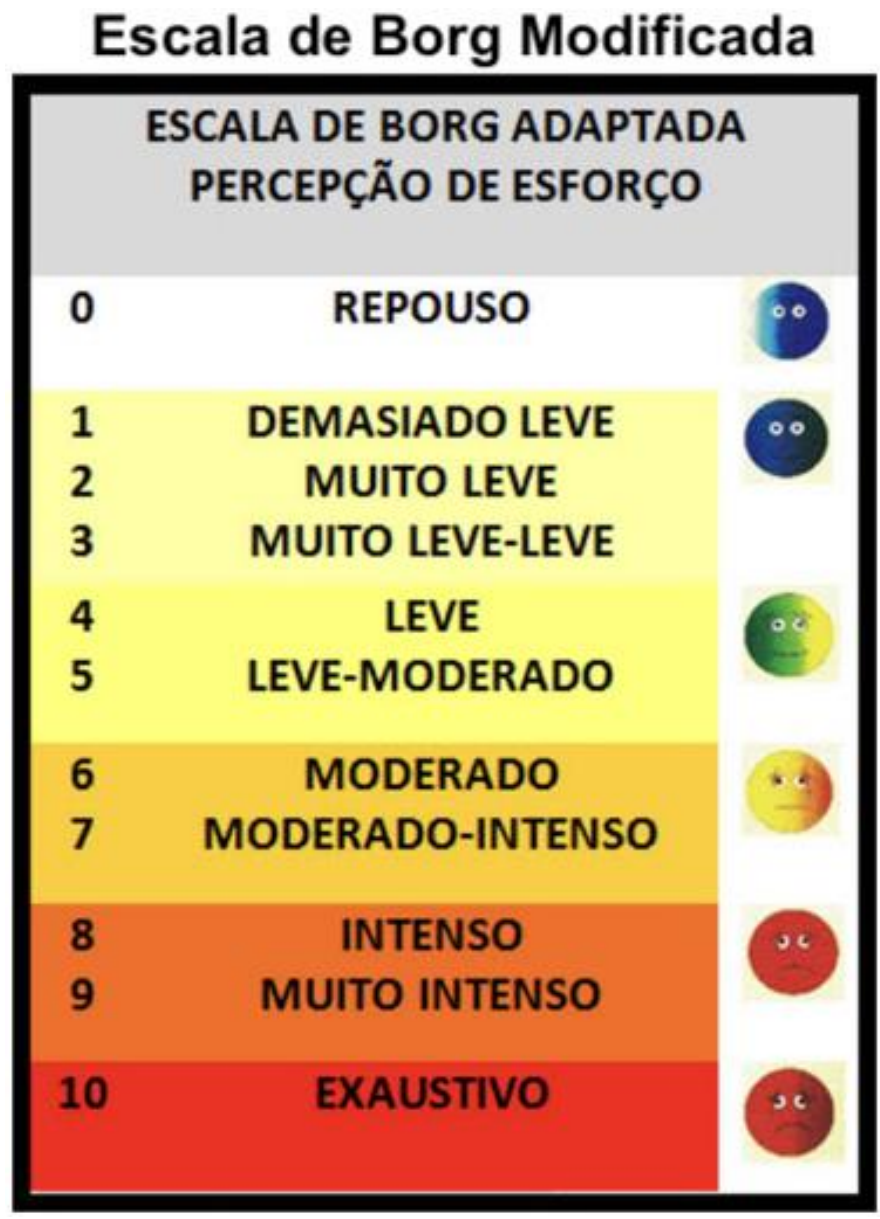

Figura 1 - Escalas de Borg modificada utilizada na avaliação pré e pós técnica de liberação miofascial instrumental. Fonte: Souza, 2017 (25).

\section{EVA}

\section{Escala de Dor}

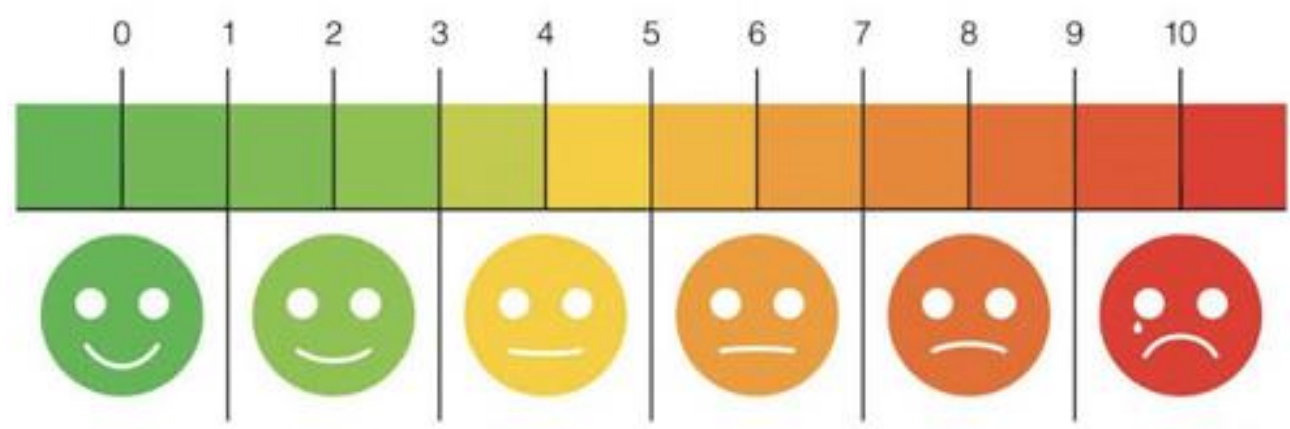

Sem Dor Dor Suave Dor Moderada Dor Forte Dor Muito Forte Dor Máxima

Figura 2 - Escala Visual Analógica utilizada na avaliação pré e pós técnica de liberação miofascial instrumental. Fonte: Adaptado de Barboza, 2019 (26). 
O atleta foi reabilitado pelo mesmo fisioterapeuta que cuidou da recuperação da cirurgia e realizou esta pesquisa. As lesões musculares foram reabilitadas com a utilização de técnicas analgésicas, na fase inflamatória (recursos eletrotermofototerápicos), seguidos na fase de reparo da lesão por cinesioterapia: resistência, força e flexibilidade muscular dos membros inferiores.

Após a recuperação da última lesão muscular o fisioterapeuta sugeriu realizar um trabalho preventivo de Liberação Miofascial, uma vez na semana, onde foi utilizado as técnicas: liberação miofascial instrumental (ventosa da marca Dong Bang; raspadores soco e guidão da marca Mioblaster; percussão com a utilização do Theragun da marca Phoenix), no período de abril a outubro/2019. O atleta continuou com a mesma programação de treinamentos e jogos semanais.

Durante a terapia, o atleta permanecia em decúbito dorsal e a ventosa era aplicada de modo estacionária com 3 sucções de vácuo e mantida por 10 minutos nos músculos quadríceps de ambos os membros inferiores; seguido da liberação com os raspadores, utilizando uma pequena quantidade de creme corporal (para facilitar o deslizamento dos equipamentos, mas não interferir na fricção dos tecidos subcutâneos); finalizando com a percussão do Theragun. Sequencialmente o atleta se posicionava em decúbito ventral e a aplicação era na mesma sequência: ventosa por 10 minutos nos isquiotibiais e gastrocnêmio bilaterais; seguido pela liberação da fáscia com os raspadores e finalizando com a percussão do Theragun. Este tratamento era realizado uma vez por semana e totalizava 60 minutos.

Os treinos de academia eram supervisionados por um personal trainer 3 vezes por semana, onde, no início a intensidade do trabalho era baixa e o volume alto (menos carga e maior número de repetições); na sequência a intensidade aumento e o volume diminuiu (menor número de repetições e carga maior); até a intensidade e o volume ficarem altos (carga alta e número de repetições maiores). A programação foi baseado nas funções de movimento corporal, subdivididos em 6 fases de treinamento. $\mathrm{O}$ atleta foi periodizado em todas elas durante o cronograma dos trabalhos: fase1 - condicionamento; fase 2 hipertrofia; fase 3 - força; fase 4 - potência; fase 5 - resistência a fadiga; fase 6 metabólicos.

As avaliações eram realizadas através da balança de bioimpedância com o Monitor de Composição Corporal Tanita Inner Scan, modelo BC-1500 plus, avaliando os percentuais de gordura e água; peso corporal e muscular. As análises eram realizadas antes dos treinamentos, a cada 10 semanas, onde o atleta ficava com o mínimo de roupa possível, subia na balança, e segurava as alças laterais, nas mãos, mantendo a posição por um tempo determinado pelo equipamento. Nos dias das avaliações a orientação era não realizar o trabalho de academia antes dos procedimentos. Os dados coletados eram enviado para um software específico vinculado ao modelo da balança.

O presente estudo foi submetido ao Comitê de Ética e Pesquisa, da Universidade de Araraquara, aprovado com número do parecer 3.893.063. O atleta foi esclarecido e orientado a respeito do objetivo e delineamento do presente estudo, conforme determina a Carta Circular 166/18 do Ministério da Saúde. Sendo assim concordou em participar e assinou o termo de consentimento livre e esclarecido.

\section{Resultados}

Os resultados compilados em questão da prática de liberação miofascial, apresentou uma melhora no quadro clínico em relação à: dores nos membros inferiores; sensação de cansaço (reavaliados pelas escalas EVA e Borg modificada) (Figura 3); lesão muscular (Figura 4). 


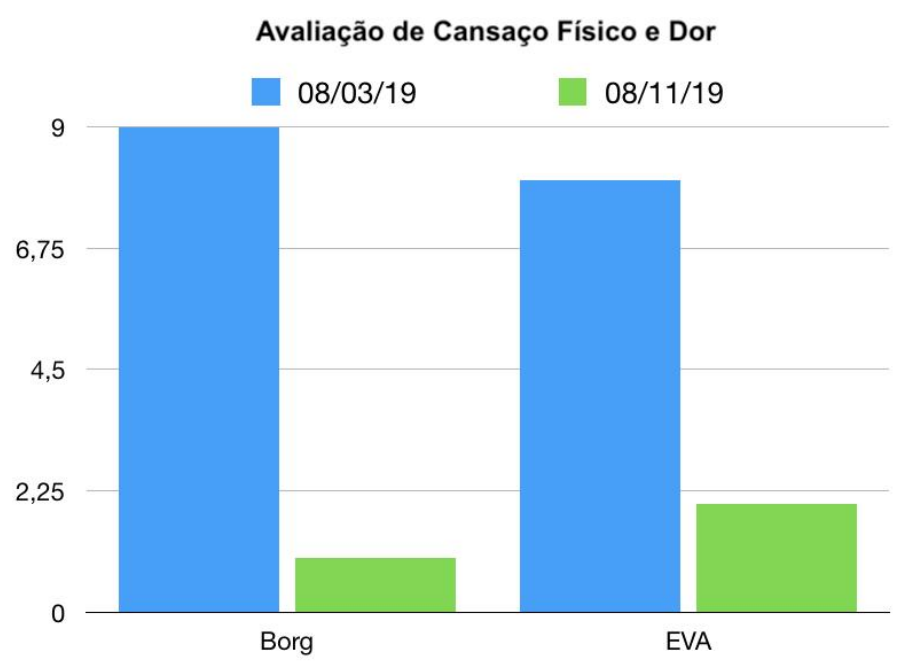

Figura 3 - Avaliação do cansaço físico e das dores musculares pré e pós intervenção da técnica.

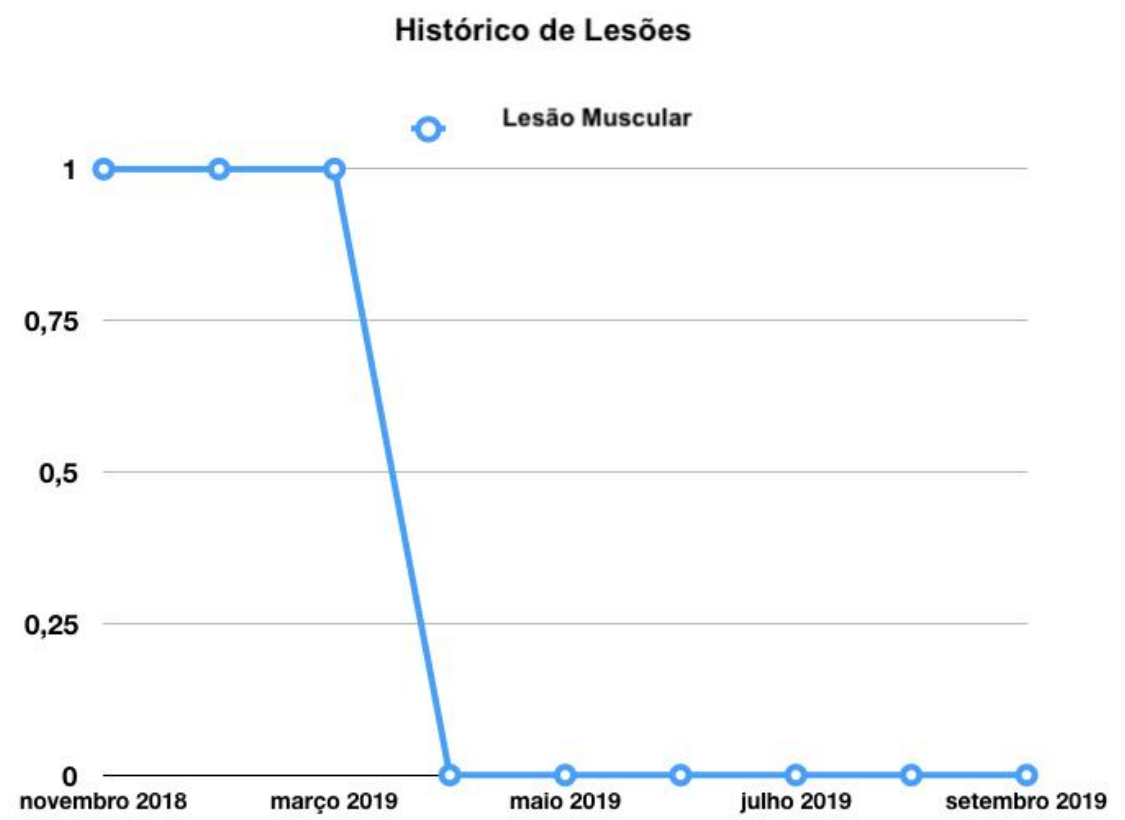

Figura 4 - Número de lesões musculares apresentados no retorno a prática esportiva e após a intervenção de liberação miofascial.

O mesmo não precisou ser afastado das suas atividades de treinamento e jogos nenhum período, apresentando até mesmo uma melhora no seu desempenho físico, e atlético.

Em relação a avaliação da composição corporal os resultados apontam os indicadores pré, pós cirurgia e lesões musculares, mostrando que o trabalho preventivo pode ter influenciado indiretamente nas questões de diminuição do percentual de gordura, e a melhora das condições físicas, uma vez que houve um aumento da massa muscular (Quadro 5). 
Quadro 5 - Percentual de gordura e água; peso corporal e muscular, pré e pós cirurgia do joelho e lesões musculares.

\section{Avaliações Físicas}

\begin{tabular}{|l|c|c|c|c|c|}
\hline & $\mathbf{0 9 / 0 1 / 1 8}$ & $\mathbf{0 9 / 0 8 / 1 8}$ & $\mathbf{2 6 / 1 0 / 1 8}$ & $\mathbf{0 4 / 0 6 / 1 9}$ & $\mathbf{0 6 / 1 1 / 1 9}$ \\
\hline Peso Total (Kg) & 97,4 & 98,7 & 99,4 & 100,7 & 97,3 \\
\hline Água (\%) & 53,8 & 53,6 & 53,1 & 52,8 & 56 \\
\hline Gordura (\%) & 22,8 & 23 & 23,7 & 24,4 & 20,1 \\
\hline $\begin{array}{l}\text { Massa Muscular } \\
\text { (Kg) }\end{array}$ & 71,5 & 72,2 & 72 & 72,3 & 73,9 \\
\hline
\end{tabular}

\section{Discussão}

O presente trabalho demonstrou o efeito da liberação miofascial na melhora da dor, na recuperação da capacidade muscular do atleta amador, consequentemente, o aumento das suas condições físicas para desempenhar as atividades, aos quais foram reavaliados através da escala de Borg modificada, EVA e bioimpedância, já citados na metodologia. Os resultados da pesquisas foram satisfatório quanto as questões observadas.

A liberação miofascial tem o objetivo de minimizar a dor muscular de início tardio, ocasionado após os treinamentos de força ou atividade exaustiva, melhorando a flexibilidade muscular, promovendo sustentação e transmissão de forças. Por um lado a tensão da fáscia é importante para coordenação do movimento, outrora, a perda de elasticidade diminui a capacidade de mobilidade ampla (27). Pode ser realizada de maneira passiva, ou ativa, aumentando do fluxo sanguíneo na região estimulada, melhorando a mobilidade deste tecido $(3,28,29)$.

O uso da terapia manual no tratamento da dor, parte do princípio que as técnicas de liberação produzem um efeito sistêmico no corpo, ocasionando uma vasodilatação, aumento do fluxo linfático, relaxamento muscular, alívio da dor, benefícios psicológicos e sensação de bem estar (30).

É uma técnica utilizada nos programas de treinamento, visando melhorar a performance antes do exercício e na recuperação da musculatura após o estresse aplicado nos treinos (3). Já alguns estudos avaliaram o efeito da auto liberação miofascial no desempenho atlético e concluíram, ser benéfica: Mc Donald e colaboradores (10), examinaram o efeito da auto liberação miofascial, na força dos extensores e na ADM do joelho. Participaram 11 indivíduos do sexo masculino (altura 178,9 $\pm 3,5 \mathrm{~cm}$, massa $86,3 \pm 7,4 \mathrm{~kg}$, idade 22,3 $\pm 3,8$ anos) divididos em grupo de aplicação e controle sem intervenção. A força do quadríceps e ADM do joelho foram mensuradas em 2 e 10 minutos antes e após a intervenção. Uma análise de variância bidirecional (condição $\times$ tempo) com medidas repetidas foi realizada em todas as variáveis dependentes registradas nos testes de pré e pós aplicação, apresentando um aumento significativo $(\mathrm{p}<0,001)$ em $10^{\circ}$ e $8^{\circ}$ de aumento da ADM. Porém antes da liberação, houve uma correlação negativa significativa $(\mathrm{p}<0,01)$ entre a força e a ADM dos indivíduos, que não apareceu mais após a prática miofascial. Isto aparenta que a técnica de liberação se beneficia do fenômeno da tixotropia, liberando as aderências, tornando o tecido conjuntivo mais maleável para a prática esportiva.

Bradbury-squires e colaboradores (12), avaliaram 10 homens (idade $=26,6 \pm 5,2$ anos, altura $=175,3 \pm 4,3 \mathrm{~cm}$, massa $=84,4 \pm 8,8 \mathrm{~kg}$ ), submetidos a 3 grupos experimentais aleatórias: G1 - 5 repetições de 20 segundos; G2 - 5 repetições de 60 segundos, de liberação miofascial com rolo; G3 - controle, sem intervenção. Avaliados com: Escala visual analógica de dor, eletromiografia do músculo vasto lateral e bíceps femoral e ADM do joelho. Os resultados não apresentaram diferença entre o tempo de aplicação de 20 e 
60 segundos. No G2 a dor foi na marca de 40 e 60 segundos apresentando 13,5\% (5,7 \pm $0,70)$ e $20,6 \%(6,2 \pm 0,70)$ respectivamente, sendo superior ao $\mathrm{G} 1$ que realizou a técnica em até 20 segundos $(\mathrm{P}<0,05)$. Em relação a ativação muscular do vasto lateral e bíceps femoral encontrou aumento de $8 \%$ e $7 \%$, respectivamente, na contração voluntária máxima e um ganho de 10 e $16 \%$ de ADM nos grupos 1 e 2, relacionados ao controle $(\mathrm{p}<0,05)$, concluindo que a liberação era dolorosa e induzia a atividade muscular, aumentando a ADM dos joelhos e a eficiência neuromuscular.

Porém Halperin e colaboradores (31), avaliaram 14 indivíduos treinados em um projeto cruzado randomizado. Após um aquecimento, os indivíduos foram avaliados quanto à ADM de dorsiflexão passiva, contração voluntária máxima e teste de equilíbrio de unilateral de olhos fechados para tornozelo, 10 minutos antes da intervenção. Seguido aleatoriamente de alongamento estático e liberação miofascial dos músculos gastrocnêmio: 3 séries de 30 segundos com 10 segundos de descanso. Após 10 minutos foram reavaliados, apresentando aumento da força no grupo da técnica fascial, com diferença significativa ( $\mathrm{p}<0,05, \mathrm{ES}=1,23$, diferença de 8,2\%). Ambos os grupos, liberação e alongamento, obtiveram um aumento da ADM imediatamente e após 10 minutos da aplicação ( $\mathrm{p}<0,05, \mathrm{ES}=0,26, \sim 4 \%$ e $\mathrm{p}<0,05, \mathrm{ES}=0,27, \sim 5,2 \%$ respectivamente).

Profissionais da área da saúde que convivem diariamente com estes atletas, recomendam este tipo de intervenção na preparação e na recuperação muscular. Vários estudos demonstram o efeito desta técnica no aumento de força, flexibilidade e performance (10). Sullivan e colaboradores (1), utilizou 7 voluntários do sexo masculino e 10 do sexo feminino, para testar a liberação miofascial na ADM de membro inferiores. Formaram 4 grupo realizando liberação nos isquiotibiais: G1 - 1 série de 5 segundos; G2 - 1 série de 10 segundos; G3 - 2 séries de 5 segundos; G4 - 2 séries de 10 segundos; grupo com 9 participantes não receberam intervenção. Foi realizado teste de sentar e alcançar, de força voluntária máxima mensurados após a liberação. Os resultados mostraram um aumento de $4,3 \%$ na ADM relacionando o tempo de execução da técnica $(\mathrm{p}<0,0001)$. Uma tendência de que a aplicação de 10 segundos leva a um ganho de ADM maior do que nos 5 segundos $(\mathrm{p}=0,069)$. Portanto não apresentou alteração significativa em relação a força, concluindo que é eficaz no ganho de ADM.

Porém Peacok e colaboradores (32), avaliaram 11 indivíduos sexo masculino num estudo cruzado divididos em 2 grupos: 1 - aquecimento total do corpo; 2 - aquecimento total do corpo e liberação miofascial. Após cada intervenção foram avaliados a flexibilidade, potência, agilidade, força e velocidade. Os dados resultaram que a associação de aquecimento e liberação melhoraram a potência, agilidade, força e velocidade comparando com o aquecimento $(\mathrm{P} \leq 0,024)$.

Skarabot (33) avaliou a efetividade do alongamento estático, liberação miofascial e a associação das duas atividades em 11 atletas na ADM passiva de dorsiflexão de tornozelo. Foi realizado aleatoriamente alongamento estático, liberação miofascial e ambas as técnicas. O teste post hoc revelou aumento na ADM pós intervenção em $6,2 \%$ no grupo de alongamento estático $(\mathrm{p}<0,05)$ e $9,1 \%$ para as técnicas associadas $(\mathrm{p}<0,05)$ e não apenas para a liberação miofascial. Contudo os 3 grupos revelaram um aumento na flexibilidade do tornozelo, porém a técnica associada é mais efetiva.

Murray e colaboradores (34) investigaram o efeito da liberação miofascial na flexibilidade e na melhora da força muscular em 12 jogadores de squash, separados em 2 grupos, sendo um deles controle. Avaliaram a flexibilidade, a força muscular dos flexores de quadril e quadríceps, além da temperatura dos músculos com o uso da termografia. Foi realizado 60 segundos de liberação miofascial e a reavaliação em 5, 10, 15 e 30 minutos. Apresentou melhora da flexibilidade do quadril e do quadríceps em 30 
minutos ( $\mathrm{p}<0,03$; aumento total de $2,4^{\circ}$ de $\mathrm{ADM}$ ), a força muscular não obteve aumento $(\mathrm{p}=0,09-0,93)$ e a temperatura não aumentou $(\mathrm{p}=0,19)$.

Su e colaboradores (35), avaliaram o efeito da auto liberação miofascial, alongamento estático e dinâmico, nos músculos quadríceps e isquiotibiais, focando a melhora do torque e flexibilidade em 15 atletas amadores do sexo masculino e 15 feminino (idade 21,43 \pm 1,48 anos, peso $65,13 \pm 12,29 \mathrm{~kg}$, altura $166,90 \pm 6,99 \mathrm{~cm}$ ). Foi avaliado o pico de torque isocinético, a flexibilidade do quadríceps pelo teste de Thomas modificado e os isquiotibiais pelo teste de sentar e alcançar. Foram realizadas 3 medidas no intervalo de 48 a 72 horas. Não encontrou diferença significativa no desempenho de força muscular, apresentando somente uma melhora significativa da flexibilidade em todos os testes.

Já Carvalho e colaboradores (3), comparou o efeito agudo da auto liberação com o alongamento estático dos músculos isquiotibiais. Foram avaliados 35 indivíduos de ambos os sexos $(16,8 \pm 1,26$ anos, estatura 168,7 $\pm 0,07 \mathrm{~cm}$ e massa corporal de $64,9 \pm$ $8,85 \mathrm{Kg}$ ) que foram divididos em dois grupos de forma aleatória: grupo alongamento estático composto por nove meninos e seis meninas; grupo auto liberação miofascial formado por 14 meninos e seis meninas. Antes e após a intervenção foi realizado o teste de sentar e alcançar para avaliar a flexibilidade. A intervenção foi nos músculos: piriforme; isquiotibiais; banda Iliotibial; quadríceps; adutores; gastrocnêmio; coluna torácica, com duração de 30 segundos por 15 de intervalo. Resultou na melhora de 10,2\% $(3,8 \pm 0,7 \mathrm{~cm})$ para o alongamento estático e melhora significativa para a auto liberação miofascial de $17,1 \%(6,1 \pm 1,5 \mathrm{~cm})$, com $(\mathrm{p}<0,05)$, ou seja, as duas técnicas melhoraram a flexibilidade dos isquiotibiais, porém a liberação foi mais eficaz. Mesmo o contexto da liberação miofascial sendo recente, a técnica está sendo implantada nos programas de treinamento visando a melhora da recuperação muscular, podendo atingir indicadores maiores na melhora de força, resistência e potência (14). Sousa (2), avaliou a eficaz da liberação miofascial na flexibilidade e força dos membros inferiores de 16 ginastas entre 13 e 16 anos, divididas aleatoriamente em um grupo experimental (auto liberação miofascial) e um controle (alongamento estático). Através dos testes: sentar e alcançar para flexibilidade; impulsão vertical e horizontal para força. As técnicas foram nos músculos piriforme, isquiotibiais, banda iliotibial, quadríceps, adutores e gastrocnêmio. Comparando as técnicas foi utilizado o test t-Student com nível de significância de p $<0,05$, obtendo uma melhora de $7 \%$ da flexibilidade, $10,5 \%$ de impulsão vertical e $10,1 \%$ no horizontal de atletas de ginástica rítmica, concluindo que a liberação miofascial influenciou na flexibilidade e na força explosiva das ginastas.

Em outra pesquisa, Lima 2018 (36), avaliou os efeitos agudos da auto liberação miofascial nos atletas da seleção de vôlei masculina, selecionando 10 atletas $(22,7 \pm 2,7$ anos; 88,6 $\pm 28,7 \mathrm{~kg} ; 169,6 \pm 39,2 \mathrm{~cm}$ ) submetidos a liberação com o Foam Roller por 60 segundos, nos músculos: tríceps sural; isquiotibiais; quadríceps. Após, percorreram duas voltas na quadra e realizaram os saltos. Os resultados mostraram: liberação miofascial (altura: 43,5 $\pm 5,75$; potência: $4118 \pm 614,3$; potência/kg: 52,9 $\pm 4,40$ ); grupo controle (altura: 42,2 $\pm 5,97$; potência: 4036,3 $\pm 653,2$; potência $/ \mathrm{kg}: 52,7 \pm 5,07$ ) não houve efeito significativo de melhora da performance, mas vale ressaltar, que os indicadores não pioraram.

Stroiney e colaboradores (37) examinaram a técnica de auto liberação com a mobilização de tecidos moles assistida por instrumento (IASTM) sobre a potência do salto vertical, horizontal e o tempo de corrida de 40 jardas de 49 estudantes universitários. Após a intervenção foi avaliado o nível de dor pela EVA e um test $t$ independente para analisar a diferenças entre as técnicas. Não houve interação $(\mathrm{p}<0,05)$ entre a intervenção da liberação e o sexo dos indivíduos, tanto no salto vertical quanto para o sprint de 40 jardas. Apresentou um efeito significativo para o salto vertical e a auto liberação $(p=0,04)$. 
O sexo também apresentou efeito significativo para o salto vertical $(\mathrm{p}=0,04)$ quanto para o sprint de 40 jardas $(\mathrm{p}=0,02)$. Não apresentou diferença significativa na percepção da $\operatorname{dor}(\mathrm{t}(49)=-1,60, \mathrm{p}>0,05)$. Portanto o uso da auto liberação miofascial antes do exercício pode melhorar a altura do salto vertical em atletas recreacionais. A dor não deve ser um fator na escolha de intervenções das técnicas para atletas porque o IASTM não foi percebido como mais doloroso do que a auto liberação. Ambas as ferramentas não melhoraram o desempenho da corrida neste estudo.

É muito importante lembrar que na fase de regeneração de uma lesão muscular pode haver uma deposição excessiva de fibras de colágeno desorganizadas (fibrose), atrapalhando os tecidos adjacentes de se mobilizarem. Portanto a liberação miofascial manual ou instrumental é uma intervenção ideal para o fisioterapeuta melhorar a mobilidade das estruturas, melhorando o trofismo muscular e facilitando o resultado de outras modalidades terapêuticas como: alongamento; outras terapias manuais; trabalho força; treinamento neuromuscular. $(38 ; 39 ; 40 ; 41 ; 42 ; 43)$.

Existe uma grande limitação da área dos estudos, onde o número de participantes das pesquisas é pequeno, tornando os resultados estreitos, onde as aplicações das técnicas restringem-se em auto liberação miofascial com rolo, sendo interessante utilizar outras maneiras como o método instrumental e a manual.

Esta técnica promove muitas questões fisiológicas importantes, restabelecendo melhorias nas condições musculares para que os atletas obtenha bons resultados.

O presente relato limitou-se somente um único caso específico. Sugestivamente, seria interessante nos estudos futuros, implementar este protocolo de liberação miofascial instrumental com um número maior de indivíduos, estudos longitudinais, limitando uma idade média e aplicando em outros esportes. Podendo estender as análises não somente na recuperação, na dor e na prevenção de lesão muscular, mas também, na influência da flexibilidade, na melhora da força e performance em atletas amadores e profissionais.

\section{Considerações Finais}

Especificamente no estudo a técnica aplicada foi eficaz na melhora recuperação muscular, da flexibilidade, da força, prevenindo lesões, uma vez que o atleta que sofria de recorrente lesões musculares, se beneficiou da aplicação, não lesionando mais suas estruturas, podendo evoluir no trabalho específico e na prática do futebol, melhorando as suas questões corporais, como: massa magra; percentual de gordura; água; peso corporal total.

Contudo pode-se considerar que a liberação miofascial instrumental, seja ela, utilizando ventosas, raspadores e percussão, atua na melhora da qualidade da musculatura esquelética, promovendo aumento da vascularização, ativação do sistema nervoso autônomo, relaxando a musculatura e melhorando a flexibilidade. Com estas alterações ocorre uma recuperação dos tecidos moles, fasciais e musculares, fazendo com que a musculatura possa desempenhar a sua função de maneira adequada obtendo melhorias do desempenho atlético.

\section{Referências}

1. Sullivan KM, Silvey DB, Button DC, Behm DG. Roller-massager application to the hamstrings increases sit-and-reach range of motion within five to ten seconds without performance impairments. Int J Sports Phys Ther 2013; 8(3): 228-236.

2. Sousa PAC, Araújo VA, Morais NA, Souza ES, Cruz RARS. Influência da autoliberação miofascial sobre a flexibilidade e força de atletas de ginástica rítmica. RPBeCS 2017; 4 (1): 18-25.

3. Carvalho LS, Araújo VA, Souza ES, Santos RMC, Mendonça WV, Arruda JRL, et al. Auto liberação miofascial x alongamento estático: efeitos sobre a flexibilidade de escolares. Rev Cen Pes Ava Qua 
Vid 2017; 9(2): 2-8.

4. Schleip R, Jäger H, Klingler W. What is 'fascia'? A review of different nomenclatures. Journal of Bodywork \& Movement Therapies 2012; 16(4): 496-502.

5. Silvini H, Antunes M, Lima VP, Silva JB, Santana H, Paz GA. Efeito agudo da técnica nos agonistas e antagonistas sobre o desempenho de repetições máximas, tempo sob tensão e percepção subjetiva de esforço na cadeira extensora. Rev Bras Pres Fis Exerc 2017; 11(69): 684-91.

6. Yeng LT, Kaziyama HHS, Teixeira MJ. Síndrome dolorosa miofascial. J Bras Ocl ATM Dor Orofa 2003; 3(9): 27-43.

7. Fernandes EHF, Fernandes JHM. Síndrome dolorosa miofascial em trabalhadores com LER/DORT. Rev Bras Med Trab 2011; 9(1): 39-44.

8. Manhein C. The Miofascial Release Manual. Thorofare: Slack Incorporated; 2001.

9. Myers, T.W. Anatomy trains: myofascial meridians for manual and movement therapists. Londres: Churchill Livingstone; 2014.

10. MacDonald GZ, Penney MD, Mullaley ME, Cuconato AL, Drake CD, Behm DG, et al. An acute bout of self-myofascial release increases range of motion without a subsequent decrease in muscle activation or force. J Strength Cond Res 2013; 27(3): 812-821.

11. Schroeder AN, Best TM. Is self myofascial release an effective pré exercise and recovery strategy? A literature review. Current Sports Medicine Reports 2015; 14(3): 200-8.

12. Bradbury-squires DJ, Noftall JC, Sullivan KM, Behm DG, Kevin E, Button DC. Roller- Massager Application to the Quadriceps and Knee-Joint Range of Motion and Neuromuscular Efficiency During a Lunge. Journal of Athletic Training 2015; 50(2): 133-40.

13. Behm DG, Chaouachi A. A review of the acute effects of static and dynamic stretching on performance. Eur J Appl Physiol 2001; 11(11): 2633-51.

14. Beardsley C, Skarabot J. Effects of self-myofascial release: A systematic review, Journal of Bodywork \& Movement Therapies 2015; 19(4): 747-58.

15. Okamoto T, Masuhara M, Ikuta K. Acute effects of self-myofascial release using a foam roller on arterial function, J. Strength Cond 2014; 28(1):69-73.

16. Silva Junior FI, Oliveira MB, Oliveira KBB, Letieri V. Efeito da liberação miofascial na flexibilidade de quadril em indicíduos praticantes de atividade física. XII Enc Ext Doc Ini Cient 2016; 3(1): 1-5.

17. Fidelis LT, Patrizzi LJ, Walsh IAP. Influência da prática de exercícios físicos sobre a flexibilidade, força muscular manual e mobilidade funcional em idosos. Rev Bras Geri Gero 2013; 16(1): 109-16.

18. Reid DA, Mcnair PJ. Passive force, angle, and stiffness changes after stretching of hamstring muscles. Medicine and Science in Sports and Exercise 2004; 36(11): 1944-48.

19. Mikkelson LO, Nupponen H, Kaprio J, Kautiainen H, Mikkelson M, Kujala UM. Adolescent flexibility, endurance strength, and physical activity as predictors of adult tension neck, lowback pain, and knee injury: a 25-year follow up study.British. Journal of Sports Medicine 2006; 40(2): 107-13.

20. Donatelli, S. A linguagem do toque: massoterapia oriental e ocidental. Rio de Janeiro: Roca; 2015.

21. Carvalho RJ, Almeida MAPT. Efeitos da massoterapia sobre o sistema imunológico. Rev Mult Psic 2018; 12(40): 353-66.

22. Costa RNV. Tratamento Conservador de uma rotura parcial do ligamento cruzado posterior no Futebol - Estudo de Caso. [Dissertação de mestrado] Escola Superior de Tecnologia da Saúde do Porto; 2016.

23. Bumeto AF. Comparação entre a escala modificada análogo visual aplicadas em pacientes com dispnéia. Rev Bras Ciênc Mov 1989; 3(1): 34-40.

24. Martinez JE, Grassi DC, Marques LG. Análise da aplicabilidade de três instrumentos de avaliação de dor em distintas unidades de atendimento: ambulatório, enfermaria e urgência. Rev Bras Reumatol 2011; 51(4): 299-308.

25. Souza JLS. Atividades físicas e exercícios físicos para pessoas com has e dm2. 2017. Disponível em: https://www.slideshare.net/jotaluiz/atividades-fsicas-e-exerccios-fsicos-para-pessoas-com-has-e-dm2.

26. Barboza VR. Intensidade da Dor - Por que Avaliar? Como Medir? 2019. Disponível em: https://victorbarboza.com.br/medindo-intensidade-da-dor/.

27. Fraga BS. Auto liberação miofascial no treinamento físico: Revisão da literatura. Porto Alegre. Monografia [Bacharel em Educação Física] Universidade Federal do Rio Grande do Sul; 2015. 
28. Cruz RARS, Santos RM, Silva FJ, Carvalho LS, Sousa PAC, Araújo VA, et al. Efeito imediato da auto liberação miofascial sobre a flexibilidade de jovens atletas. Arch of Spor Scien 2017; 5(2): 30-33.

29. Silva DL, Monteiro ER, Neto VGC, Triani FS. Efeitos da liberação miofascial sobre a flexibilidade: uma revisão sistemática. J Heal Sci 2017; 19 (2):200-4.

30. Araújo ACP, Felber DT, Yamada EF, Teixeira LP, Silva MD. Corrente interferencial e terapia manual reduzem sinais e sintomas de dos lombar crônica. Rev Cienci Sau Vitta 2018; 30(2): 9-20.

31. Halperin I, Aboodarda SJ, Button DC, Andersen LL, Behm DG. Roller massager improves range of motion of plantar flexor muscles without subsequent decreases in force parameters. The Inter Jour of Spor Physi Ther 2014; 9 (1):92-102.

32. Peacock CA, Krein DD, Silver TA, Sanders JG, Kyle-Patrick A, Carlowitz V. An acute bout of selfmyofascial release in the form of foam rolling improves performance testing. Int J Exerc Sci 2014; 7(3): 202-11.

33. Skarabot J, Beardsley C, Stirn C. Comparing the effects of self-myofascial release with static stretching on ankle range-of-motion in adolescent athletes. The International Journal of Sports Physical Therapy 2015; 10(2): 203-12.

34. Murray AM, Jones TW, Horobeanu C, Turner AP, Sproule J. Sixty seconds of foam rolling does not affect functional flexibility or change muscle temperature in adolescent athletes. Int J Sports Phys Ther 2016; 11(5): 765-76.

35. Su H, Chang NJ, Wu WL, Guo LY, Chu H. Acute effects of foam rolling, static stretching, and dynamic stretching during warm-ups on muscular flexibility and strength in young adults. Jour of Spo Rehabil 2017; 26(6): 469-77.

36. Lima CVAA. O efeito agudo da auto liberação miofascial na potência do salgo vertical em atletas de vôlei da UFRN. Monografia [Educação Física] Universidade Federal do Rio Grande do Norte; 2018.

37. Stroiney DA, Mokris RL, Hanna GR, Ranney JD. Examination Of Self-Myofascial Release Vs. Instrument-Assisted Soft-Tissue Mobilization Techniques On Vertical And Horizontal Power In Recreational Athletes. The Jour Stren And Conditi Rese 2018; 34 (1): 79-88.

38. Barra ME, López C, Fernández G, Murillo E, Villar E, Raya L. The immediate effects of diacutaneous fibrolysis on pain and mobility in patients suffering from painful shoulder: A randomized placebocontrolled pilot study. Clin Rehabil 2011; 25(4): 339-48.

39. Salem W. Influence de la technique de fibrolyse diacutanée sur la rotation latérale passive de l' articulation gléno-humérale Influence de la technique de fibrolyse diacutanée sur la rotation de l' articulation gléno-humérale. La Rev l'Ostéopathie 2012; 2(2): 5-14.

40. Seco Calvo J, Alonso-Cortés B, García Gil M, Gómez Jiménez M, González Sánchez M, Caro Puértolas B. Métodos específicos de intervención en fisioterapia. Madrid: Editorial Médica Panamericana; 2016.

41. Geyt B Van, Dugailly P-M, Sobczak S. Fibrolyse myofasciale par technique de crochetage. París: Éditions Conaissances et Savoirs; 2017.

42. López CC, Barra LME, González RV, Domínguez CA, Fanlo MP. Efectos a corto y medio plazo de un programa de Fisioterapia que incluye fibrolisis diacutánea en la epicondilalgia lateral crónica. Ensayo clínico no controlado. Cuest Fisioter 2017; 46 (1):12-22.

43. Arbonês AM. Efectividad de la aplicación de un protocolo de fibrolisis instrumental miofascial sobre el aumento en la fuerza máxima de la musculatura del antebrazo. ensayo clínico controlado aleatorizado [Monografia] Facultad De Enfermería Y Fisioterapia; 2018. 
Vittalle - Revista de Ciências da Saúde v. 32, n. 1 (2020) 223-234

\section{EDITORA E GRÁFICA DA FURG}

CAMPUS CARREIROS

CEP 96203900

editora@furg.br 